\title{
AN INTRAOCULAR PRESSURE SENSOR BASED ON A GLASS REFLOW PROCESS
}

\author{
R. M. Haque and K. D. Wise
}

Engineering Research Center for Wireless Integrated MicroSystems (WIMS)

The University of Michigan, Ann Arbor, MI 48109-2122 USA

\begin{abstract}
This paper presents an intraocular pressure sensor fabricated using a new process that reflows glass into DRIE-etched silicon cavities to form three-dimensional microstructures. The capacitive pressure sensor is formed using a $4 \mu \mathrm{m}$ thick boron etch-stopped silicon diaphragm having dimensions of $0.85 \mathrm{~mm} \times 1.65 \mathrm{~mm}$. The sensor operates in touch-mode with a linear response and sensitivity of $26 \mathrm{fF} / \mathrm{mmHg}$, resolving better than $1 \mathrm{mmHg}$ over a $650-850 \mathrm{mmHg}$ dynamic range. Vertical silicon feedthroughs in the glass provide electrical access to the sensor. This pressure sensor is part of an intraocular microsystem being developed for monitoring the eye pressure of patients diagnosed with glaucoma.
\end{abstract}

\section{INTRODUCTION}

Glaucoma is the second-leading cause of blindness, affecting over 68 million people worldwide. Intraocular pressure is important in detecting and treating this disease, but its measurement involves equipment typically found in a hospital or doctor's office, limiting the frequency of measurements and complicating treatment. A number of implantable intraocular pressure sensors have been reported recently [1-3], but many of the reported devices have been excessively invasive or may be large enough to partially occlude the field of view in low-light situations. A compact, highly-integrated intraocular microsystem (Fig. 1) is being developed that will record pressure every 15 minutes, transmitting the stored data to the outside world once a day on demand. The microsystem will contain an ultra-low-power microcontroller [4], power source, capacitive pressure sensor, and wireless interface, all housed in a parylene-coated glass package. The use of a glass substrate ensures sensor hermeticity, enhances antenna performance, and allows photovoltaic energy scavenging. Vertical feedthroughs in the glass provide electrical connections to the pressure sensor and to the antenna, which are mounted on the external surfaces of the intraocular package.

The pressure sensor component of the full microsystem is described in this work, with a specific focus on a newly developed fabrication technique. Boron doping defines the diaphragm thickness, while vertical interconnects made of highly-doped silicon provide electrical connections to the plates of the capacitive sensor. The pressure sensor exhibits a linear response when operating in touch-mode, where the sensor is constantly in contact with the bottom plate. Changes in pressure change the contact area rather than gap, resulting in the linear response. While stiction effects are present, they do not significantly degrade the required resolution of the device. The vacuum-referenced sensor operates with a dynamic range of $650 \mathrm{mmHg}$ to $850 \mathrm{mmHg}$ to accommodate changes in ambient pressure, such as pressurized cabins of an airplane or travel to higher elevations. The external readout module will record atmospheric pressure and compensate for these changes when the intraocular pressure is transferred from the implanted microsystem. Intraocular pressure typically varies between 8 and $50 \mathrm{mmHg}$, with the onset of glaucoma defined as a pressure value above $21 \mathrm{mmHg}$ relative to ambient. A resolution of $1 \mathrm{mmHg}$ is used for standard tonometric methods, such as Goldmann applanation, in the doctor's office.

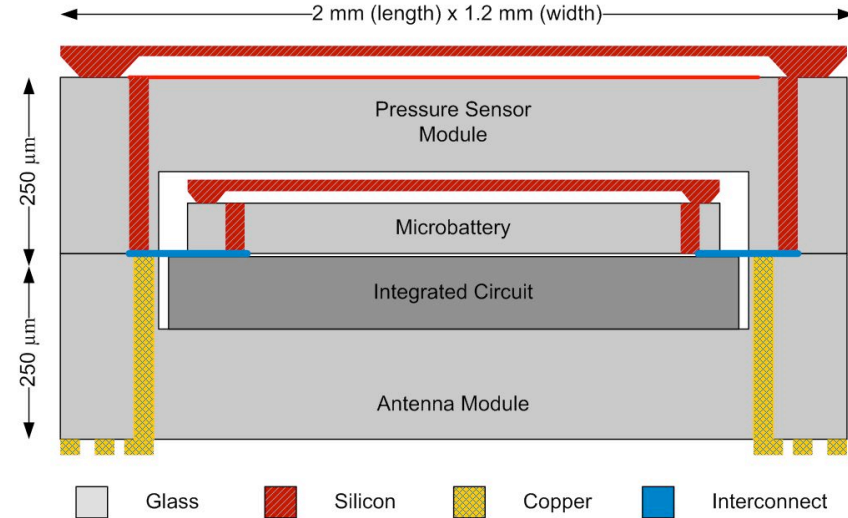

Fig. 1: A complete intraocular microsystem, demonstrating vertical interconnects and glass packaging of the components.

Accessing the internal plate of hermetically-sealed capacitive pressure sensors is an important issue in designing these devices, and in the past, such sensors were implemented using lateral feedthroughs employing polysilicon or eutectic metal access leads [5-6]. The device used in this work uses vertical connections through the glass to simplify electrical access to the capacitor and keep the leads totally within the sealed implantable package. This also helps minimize the overall size of the device.

\section{FABRICATION PROCESS \\ Glass Reflow}

Since glass is difficult to micromachine with precision, a new wafer-level glass-in-silicon process (Fig. 2) has been developed. In this process, a highly-doped silicon wafer (p-type, $<0.01 \Omega$-cm) is etched $250 \mu \mathrm{m}$ deep using DRIE, forming a mold. After anodically bonding in vacuum (Suss SB6e) to a standard $500 \mu \mathrm{m}-$ thick borosilicate glass wafer (Plan Optik), the silicon-glass sandwich is heated above the glass transition temperature, causing the glass to reflow into the mold as shown in Fig. 2, step 2. Optimization of the reflow temperature, reflow time, and mask design rules ensures appropriate glass filling and flow. For this process, heating above $750^{\circ} \mathrm{C}$ for a period of 8 hours was sufficient to achieve complete glass filling, as shown in Fig. 3.

1

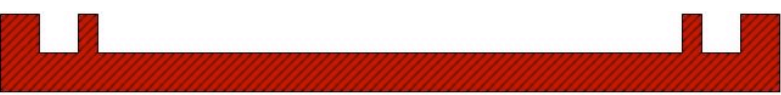

2

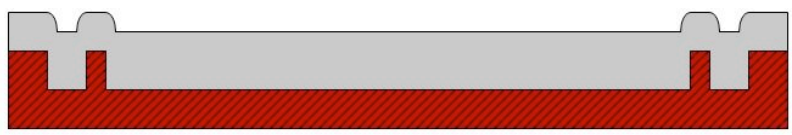

3

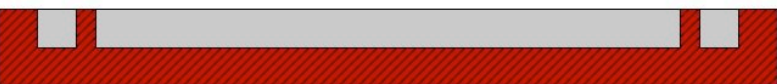

4 Glass Silicon Aluminum Fig. 2: Process flow for forming glass-in-silicon wafers with integrated feedthroughs. 


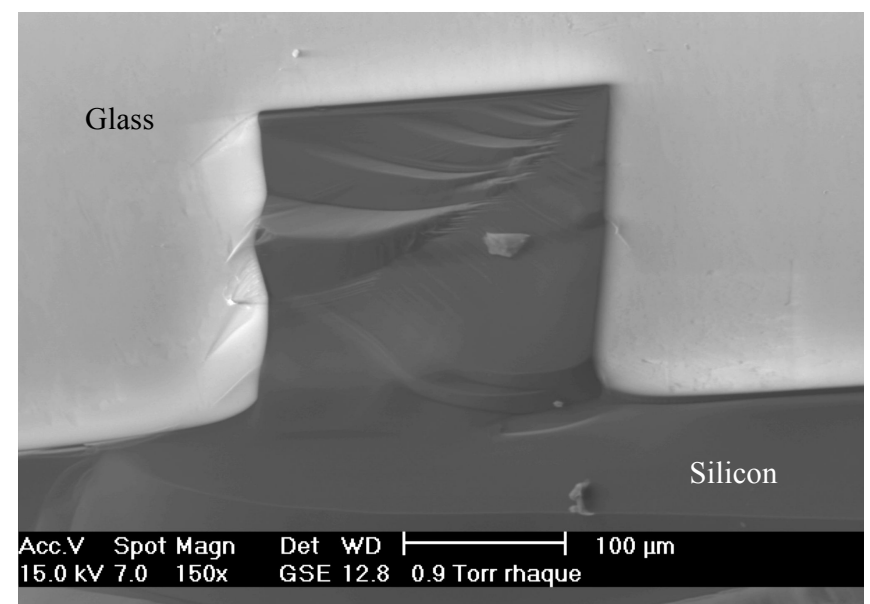

Fig. 3: Cross-section of the glass reflow process showing complete glass filling of a cavity in the silicon. The lighter colored region is glass, while the lower, darker region, is silicon.

The planarization and polishing shown in Fig. 2, step 3, was accomplished with an IPEC chemical mechanical polishing (CMP) tool with double platens. One platen is dedicated to conventional polishing with slurry while the other platen was retrofitted with a special diamond-embedded pad (Trizact, 3M). Lapping on the diamond pad planarizes the glass until it is flush with the surface of the silicon. The surface can then be polished on the other pad while flowing slurry specifically designed for oxides. The silicon is simultaneously polished in this step as well, although at a slightly slower rate than the glass. For later process steps such as anodic bonding, this polishing step is absolutely critical. Wafers that have some surface roughness or high spots will not adequately bond, and any roughness can serve as a source of potential leaks.

In the final processing step, the silicon is lapped from the back side in a similar fashion to the process used on the front side until the glass regions are exposed. A second CMP step can be used to polish the back side of the wafers if double-sided polished glass-in-silicon wafers are desired. Mounting the wafer on a carrier is essential for this thinning step.

Although similar processes have been reported [7-8], this is the first time such a glass-in-silicon process is thought to have been demonstrated at the wafer level. The completed glass-insilicon wafer can be further processed using conventional fabrication techniques. Although the $100 \mathrm{~mm}$ wafers are only $250 \mu \mathrm{m}$ thick after thinning, they are robust enough to survive conventional spin-rinse driers and process steps such as liftoff. Fig. 4 shows a completed $100 \mathrm{~mm}$ glass-in-silicon wafer.

\section{Pressure Sensor Design and Fabrication}

The capacitive pressure sensor is fabricated using the dissolved-wafer process as shown in Fig. 5. The sensor is designed with a nominal $15 \mu \mathrm{m}$ zero-pressure gap, defined using a $\mathrm{KOH}\left(45 \%\right.$ at $\left.85^{\circ} \mathrm{C}\right)$ recess etch into a standard p-type silicon wafer (Fig. 5, step 1). Two boron-doping steps are then used to produce etch-stops that set the device dimensions. A deep boron diffusion sets the eventual rim thickness at $15 \mu \mathrm{m}$. This is followed by a shallow boron diffusion that sets the diaphragm thickness at $4 \mu \mathrm{m}$. The lateral dimensions of the diaphragm are lithographically set at $0.85 \mathrm{~mm} \times 1.65 \mathrm{~mm}$. A $150 \mathrm{~nm}$-thick silicon dioxide layer is thermally grown over the surface of the diaphragm to protect against shorts when the plates touch. As is well known, the pressure sensitivity of capacitive pressure sensors is a strong function of the gap spacing, diaphragm thickness, lateral dimensions, and internal diaphragm stress [9]. The lateral dimensions are controlled to better than $1 \mu \mathrm{m}$ by lithography, while the diaphragm thickness is set by the etch-stop to within about $\pm 0.2 \mu \mathrm{m}$. Diaphragm stress is also highly reproducible with the use of a thermal oxide adding compressive stress to the diaphragm. A modified oxide-nitride stack or an oxynitride film could also be used to set the stress as desired. The gap is more widely variable, however, and as in most pressure sensors, individual calibration is required to meet the demands of the intraocular application. For this system, this will be accomplished in the embedded processor of the intraocular microsystem.

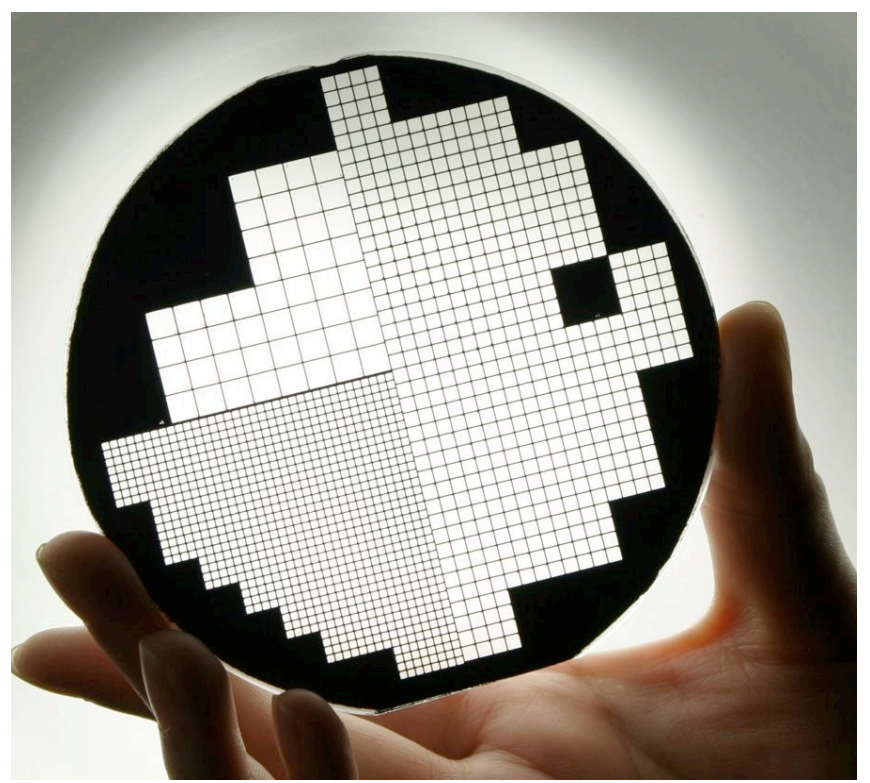

Fig. 4: A completed glass-in-silicon 100mm-diameter wafer.
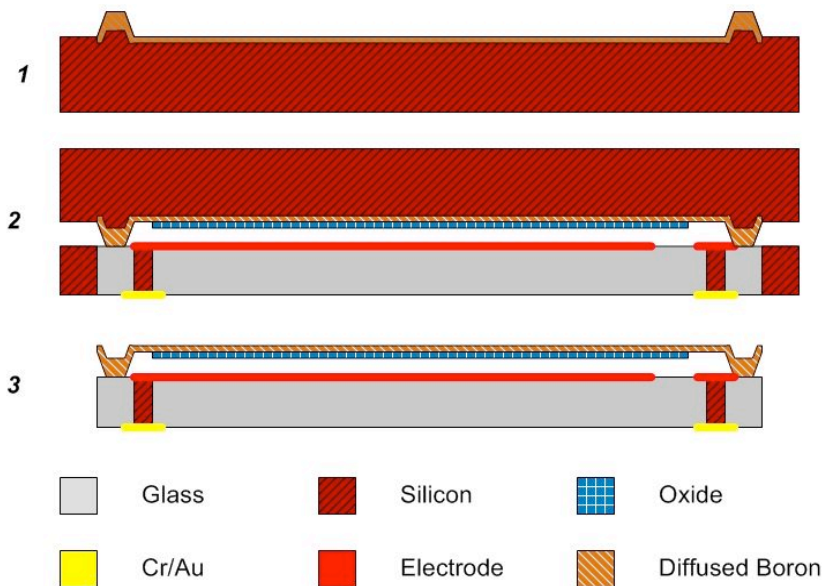

Fig. 5: Dissolved-wafer process flow for the intraocular pressure sensor. Step 2 shows the bonding of a silicon diaphragm wafer with a glass-in-silicon wafer.

The glass-in-silicon wafer serves as the substrate to which the diaphragm rim is bonded. A thin stack of titanium, platinum, and gold is patterned using liftoff to serve as the electrical contact within the pressure sensor cavity. Each vertical silicon feedthrough is electrically connected to the two different capacitive plates in this single masking step. One forms the bottom electrode on the glass surface, while the other provides a connection to the highlydoped boron diaphragm, forming the upper electrode. 
With the diffusions and the diaphragm oxide in place, the diaphragm wafer and the glass-in-silicon wafer are aligned and anodically bonded (Suss MA-6/BA-6 and SB6e) together using a $1.5 \mu \mathrm{m}$-thick aluminum sacrificial spacer (on the glass-in-silicon wafer, as shown in Fig. 2, step 4) to distribute the electrical field and prevent unintended shorts. After bonding, a chromium/gold stack (Fig. 5, step 2) is deposited and defined using liftoff to make ohmic contacts to the feedthroughs and protect them during subsequent processing. The wafers are then placed in an ethylenediamine pyrocatechol (EDP) etch to remove the lightlydoped silicon and perform die separation (Fig. 5, step 3). The overall die size is approximately $1.2 \mathrm{~mm} \times 2 \mathrm{~mm}$. The glass is $250 \mu \mathrm{m}$ thick and provides appropriate interconnects to the sensing plates for the capacitor. Fig. 6 shows several completed intraocular pressure sensors on a U.S. penny, while Fig. 7 shows an SEM profile view of one of the devices.

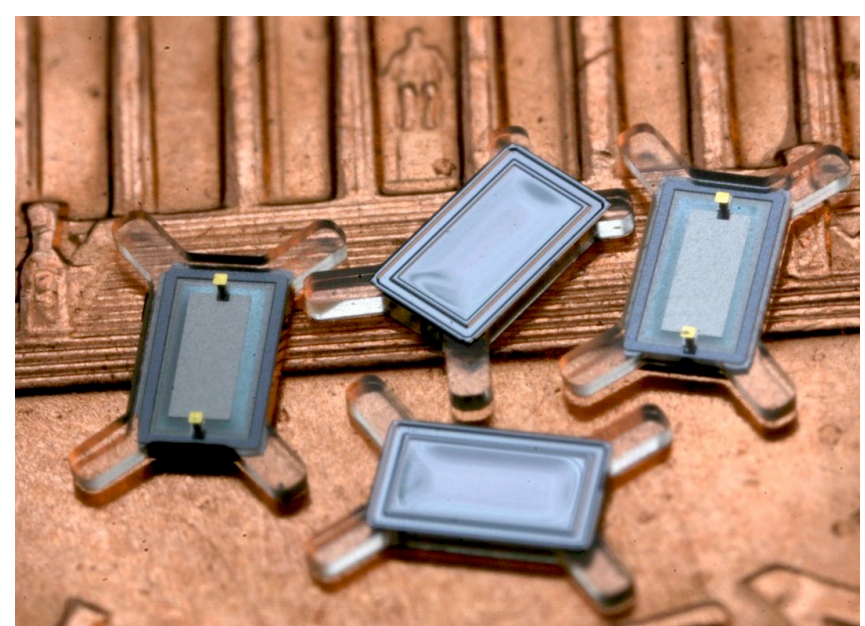

Fig. 6: Completed intraocular pressure sensors on a US penny, showing the electrical contacts on the back of the devices and the vacuum-sealed silicon diaphragm on the front. The diaphragm size is $0.85 \mathrm{~mm} \times 1.65 \mathrm{~mm}$.

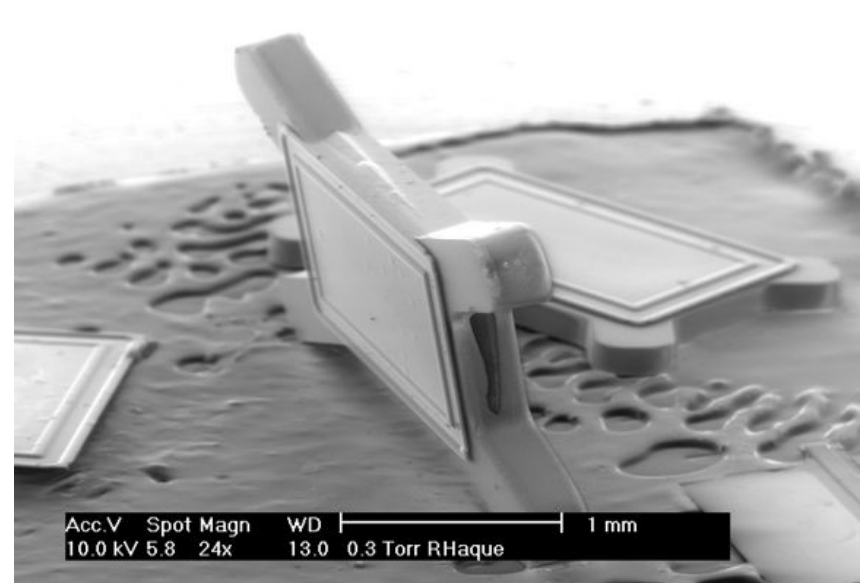

Fig. 7: An SEM view showing the profile of a released pressure sensor. The silicon sensor rim is $15 \mu \mathrm{m}$ thick, and supports a $4 \mu \mathrm{m}$ thick diaphragm. The glass is approximately $250 \mu \mathrm{m}$ thick.

\section{PRESSURE SENSOR PERFORMANCE}

The pressure sensors were designed and simulated using COMSOL to estimate appropriate diaphragm dimensions. Depending on the dimensions chosen, the device can operate in either the typical non-touching (gap-sensitive) mode or in the touching (area-sensitive) mode. In either case, the glass substrate serves as an overpressure stop. Fig. 8 shows the simulated deflection for diaphragm thicknesses of $4 \mu \mathrm{m}$ and $5 \mu \mathrm{m}$, ignoring any overpressure stop. The large variation in deflection with only a one-micron change in diaphragm thickness is quite clear.

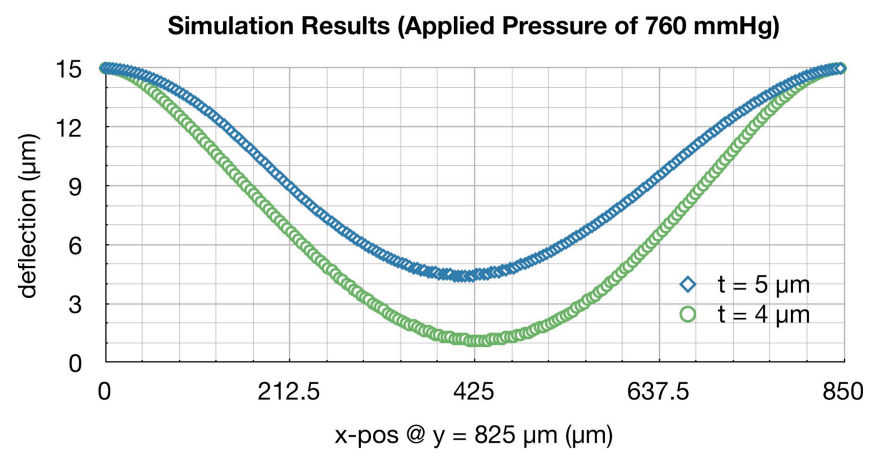

Fig. 8: Simulated deflection of a $0.85 \mathrm{~mm} \times 1.65 \mathrm{~mm}$ diaphragm for two values of diaphragm thickness. No overpressure stop is included.

All capacitance measurements were made using an HP4284A LCR meter operating with a sampling rate of $1 \mathrm{MHz}$. The silicon diaphragm serves as one plate of the capacitance with the electrode on the glass as the other plate. Fig. 9 shows the overall measured capacitance versus pressure characteristic for pressures below an atmosphere. The two regions of operation are clearly visible. Below about $450 \mathrm{mmHg}$ the plates do not touch and the response is highly non-linear, characteristic of gap-sensitive sensors. Above this level, the plates touch, and the response becomes linear.

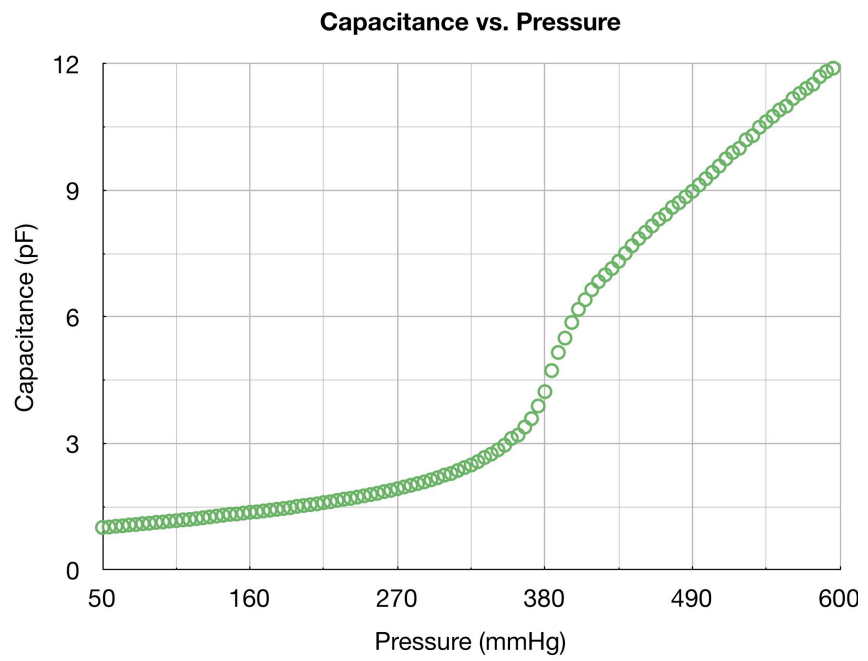

Fig. 9: Measured pressure versus capacitance characteristic of an intraocular pressure sensor, showing touch-mode operation above about $450 \mathrm{mmHg}$.

The capacitance versus pressure characteristic was next measured in an externally-regulated pressure chamber. In order to evaluate the possible effects of stiction, the applied pressure was swept from $750 \mathrm{mmHg}$ down to $550 \mathrm{mmHg}$, then slowly increased to $850 \mathrm{mmHg}$, and then returning again to $750 \mathrm{mmHg}$. Although some hysteresis can be detected, presumably due to stiction at the interface between the oxide-coated diaphragm and the sensing electrode on the glass, after correction the estimated maximum error due to these effects should fall within the $1 \mathrm{mmHg}$ resolution 
required in this application. Fig. 10a shows the response of the pressure sensor with a detail view around $750 \mathrm{mmHg}$ shown in the plot of Fig. 10b. The pressure sensitivity is $26 \mathrm{fF} / \mathrm{mmHg}$.

Capacitance vs Pressure Sweeps (750 to 550 to 850 to $750 \mathrm{mmHg}$ )
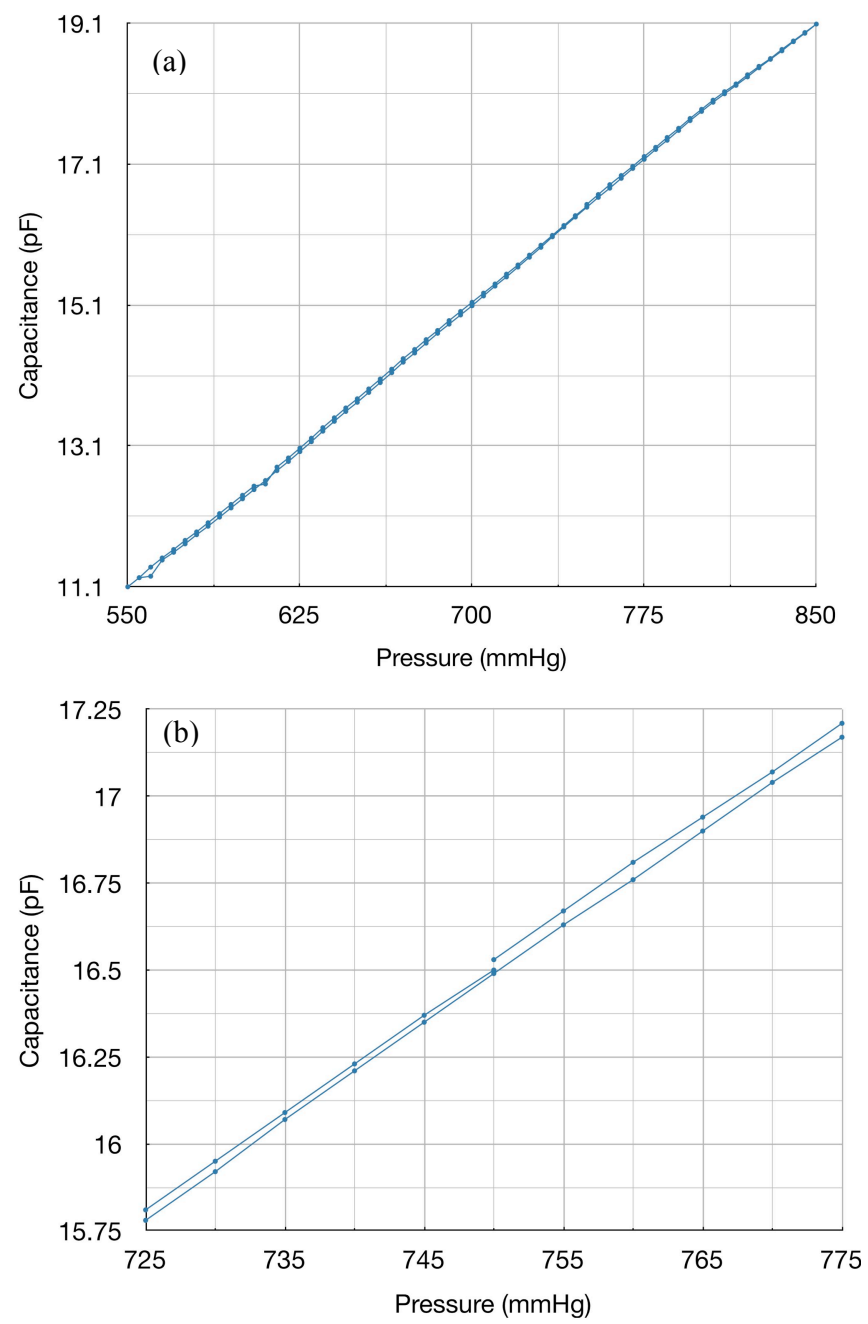

Fig. 10: (a) Measured capacitance versus pressure while cycling pressure from 750 to 550 to 850 and back to $750 \mathrm{mmHg}$; (b) closeup view around $750 \mathrm{mmHg}$.

The $100 \mu \mathrm{m}$-diameter silicon feedthrough resistances were measured to be about $2.2 \Omega$, in good agreement with the calculated resistance of approximately $3.2 \Omega$ for $0.01 \Omega-\mathrm{cm}$ bulk resistivity silicon. Fig. 11 is a photo of a glass-in-silicon wafer used for electrical testing. These wafers have electrical pads on one side of the wafer and metal interconnects in various patterns on the other.

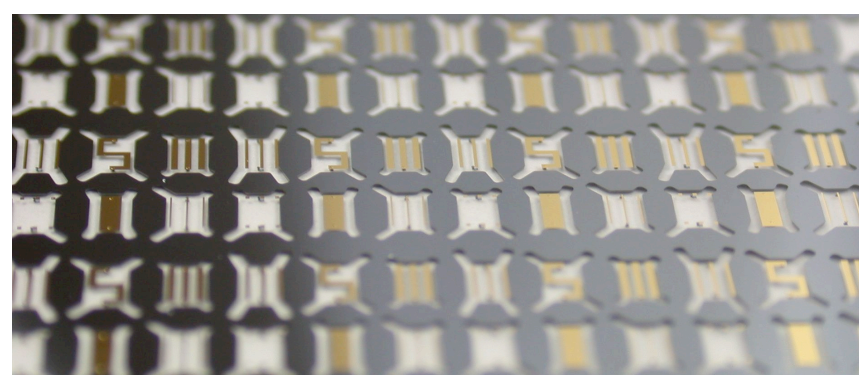

Fig. 11: A photo of die used for electrical testing of feedthroughs.

\section{CONCLUSION}

This paper presents a new process for creating high-aspectratio glass microstructures and has demonstrated the process with the design and fabrication of a pressure sensor for an intraocular microsystem. The pressure sensor operates in touch-mode with a sensitivity of $26 \mathrm{fF} / \mathrm{mmHg}$ and linear response. Hysteretic effects are present, but deemed acceptable for the intraocular application and the expected operation of the microsystem.

The simple and robust glass-in-silicon process may be useful in a wide range of new devices, especially those benefiting from a transparent substrate, vertical feedthroughs, electrical isolation, thermal isolation, or complex die shapes. The process is currently being used for several aspects of the intraocular microsystem, including the pressure sensor, the embedded microbattery, and the antenna.

\section{ACKNOWLEDGEMENTS}

The work described here was supported primarily by the Engineering Research Centers Program of the National Science Foundation under Award Number ECE-9986866 and by a gift from Ms. Polly Anderson.

\section{REFERENCES}

[1] P-J. Chen, D. C. Rodger, S. Saati, M. S. Humayun, and Y-C Tai, "Microfabricated Implantable Parylene-Based Wireless Passive Intraocular Pressure Sensors," IEEE J. Microelectromech. Syst., 17, pp. 1342-1351, December 2008.

[2] K. Stangel, S. Kolnsberg, D. Hammerschmidt, B. J. Hosticka, H. K. Trieu, and W. Mokwa, "A Programmable Intraocular CMOS Pressure Sensor System Implant," IEEE J. Solid-St. Circuits, 36, pp. 1094-1100, July 2001.

[3] E. Y. Chow, S. Chakraborty, W. JU. Chappell, and P. P. Irazoqui, "Mixed-Signal Integrated Circuits for SelfContained Sub-Cubic Millimeter Biomedical Implants," IEEE Int. Sol-St. Circuits Conf., pp. 236-237, February 2010.

[4] M. Seok, S. Hanson, Y.-S. Lin, Z. Foo, D. Kim, Y. Lee, N. Liu, D. Sylvester, and D. Blaauw, "The Phoenix Processor: A 30pW Platform for Sensor Applications," IEEE Symp. On VLSI Circuits, pp. 188-189, 2008.

[5] A. V. Chavan and K. D. Wise, "Batch-Processed VacuumSealed Capacitive Pressure Sensors," IEEE Journal of MicroElectroMechanical Systems, pp. 580-588, December 2001.

[6] A. DeHennis and K. D. Wise, "An All-Capacitive Sensing Chip for Absolute Pressure, Temperature, and Relative Humidity," IEEE International Conference on Solid-State Sensors and Actuators (Transducers '03), Boston, pp. 18601863, June 2003.

[7] P. Merz, H. J. Quenzer, H. Bernt, B. Wagner and $M$. Zoberbier, "A Novel Micromaching Technology for Structuring Borosilicate Glass Substrates," IEEE Int. SolidState Sensors and Actuators Conf., 2003, pp. 258-261.

[8] C-W. Lin, et al., "Implementation of Silicon-on-Glass MEMS Devices with Embedded Through-Wafer Silicon Vias using the Glass Reflow Process for Wafer-Level Packaging and 3D Chip Integration", J. Micromech. Microengr., vol. 18, 2008.

[9] S. T. Cho, K. Najafi, and K. D. Wise, "Internal Stress Compensation and Scaling in Ultrasensitive Silicon Pressure Sensors," IEEE Transactions on Electron Devices, 39, pp. 836-842, April 1992.

\section{CONTACT}

R. M. Haque: rhaque@umich.edu 